Ann. Zootech., I977, 26 (I), I5-28.

\title{
Utilisation des protéines par le veau préruminant à l'engrais V. - Utilisation digestive des protéines du poisson, du soja et de la féverole
}

\author{
P. Guilloteau, R. TOUllec, J. Culioli (1) et D. LE DOUARon* \\ avec la collaboration technique de \\ Marguerite Beaufils, J. Lareynie et Yvette Lento \\ Station de Recherches zootechniques, \\ * Laboratoire de Recherches de Technologie Laitière, \\ Centre de Recherches de Rennes, I.N.R.A. \\ 6.5, rue de Saint-Brieuc \\ 35042 Rennes Cedex (France)
}

\section{Résumé}

Le but de ce travail a été d'étudier chez le veau préruminant, l'utilisation digestive de différentus protéines susceptibles de remplacer celles du lait dans les aliments d'allaitement.

Quatre concentrés protéiques ont été utilisés. L'un d'entre eux (Poisson micronisé) était une farine de poisson de Norvège délipidée à l'hexane et finement broyée. Deux des autres étaient des tourteaux de soja débarrassés de leur fraction oligosaccharidique par fermentation par Geotrichum candidum (Soja fermenté) ou par extraction éthanolique à chaud (Soja concentré). Le quatrième (Féverole) a été obtenu à partir de graines de féverole par extraction aqueuse à $\mathrm{pH} 8,5$. Ces concentrés protéiques ont servi à préparer quatre aliments d'allaitement (aliments Poisson micronisé, Soja fermenté, Soja concentré et Féverole) dans lesquels ils apportaient respectivement 8I, 77, $7^{8}$ et $73 \mathrm{P}$. Ioo des matières azotées, le reste étant fourni par du lactosérum et un supplément de méthionine.

Douze veaux mâles ( 3 par régime) ont été utilisés. Ils ont été maintenus en cages à bilans entre les âges d'environ 8 et 54 à 96 jours. L'appétit, la croissance et l'état sanitaire ont été beaucoup plus médiocres avec l'aliment Féverole qu'avec les autres aliments.

Au delà du premier mois de vie des animaux, la digestibilité des matières azotées a été la plus élevée pour l'aliment Poisson micronisé $(85,5$ p. Ioo); elle a été moins satisfaisante pour les aliments Soja concentré $(82,8$ p. Ioo) et Soja fermenté $(80,0$ p. Ioo) et très faible pour l'aliment Féverole $(74,9$ p. 100). Les valeurs estimées pour la digestibilité des protéines du Poisson micronisé, du Soja concentré, du Soja fermenté et de la Féverole sont respectivement de 83, 8o, 76 et 67 p. ıoo. La rétention de l'azote apparemment absorbé a été satisfaisante pour tous les régimes $(55$ à 63 p. 100).

Sous réserve d'essais complémentaires, les concentrés protéiques de poisson et de soja étudiés pourraient donc être incorporés en quantité importante dans les aliments d'aliaitement. En revanche, il n'en serait pas de même pour le concentré de féverole, sans une amélioration de sa technologie de préparation. 


\section{Introduction}

Nous avons entrepris l'étude de l'utilisation de différentes sources de protéines susceptibles d'être substituées au lait écrémé dans les aliments d'allaitement destinés au veau préruminant à l'engrais (Toullec et al., I969; Toullec, Thivend et Mathieu, I97I; Paruelle et al., i972; Frantzen et al., i973; Toullec, Mathieu et Pion, I974; Paruelle et al., I974). Les résultats rapportés ici concernent l'utilisation digestive de concentrés protéiques de soja, de féverole et de poisson. Ces sources de protéines ont des compositions en acides aminés intéressantes (PION, I97I). Cependant, l'incorporation de tourteau de soja cru dans les aliments d'allaitement (Shoptaw, r936; Williams et KnodT, I950; Noller et al., I956; LASSister et al., I959) a conduit à des résultats médiocres qui sont dus notamment à la présence de facteurs antitrypsiques dans ce tourteau. Ces facteurs réduisent les sécrétions et l'activité de la trypsine et de la chymotrypsine (GoRRILL et al, , I967), limitant ainsi la protéolyse intestinale (GoRRILL et Tromas, I967). Le traitement du tourteau par la chaleur humide détruit les facteurs antitrypsiques et améliore l'utilisation digestive de ses protéines (NITSAN et al., I97I). L'élimination de la majeure partie des glucides permet également d'accroître l'utilisation digestive (PorTER et HILl, I963). Différents traitements technologiques peuvent être envisagés pour éliminer les glucides; dans notre essai, nous avons étudié l'utilisation de deux concentrés protéiques obtenus l'un par fermentation et l'autre par extraction éthanolique des $\alpha$ galactosides à chaud.

L'utilisation des protéines du poisson et surtout de la féverole n'a fait l'objet que d'un nombre limité de travaux chez le veau préruminant. Les protéines de la farine de poisson présentent une utilisation digestive satisfaisante ( 84 à 88 p. Ioo, d'après Van Hellemond, I967 et Van WeERDen, I974); leur hydrolyse partielle améliore leur digestibilité qui peut alors atteindre 9I p. IOo (PARUELle et al., I974). TERroIne (I93I) a substitué à une partie du lait entier, un lait de remplacement contenant de la farine de féverole; il a alors observé des diarrhées qui l'ont amené à arrêter l'expérience. Plus récemment, Duthie et al. (I974) ont obtenu une croissance satisfaisante chez le veau et l'agneau en remplaçant environ $40 \mathrm{p}$. Ioo des protéines du lait par celles d'un isolat de féverole. Dans notre essai, nous avons étudié l'utilisation d'une farine de poisson délipidée à l'hexane, ainsi que celle d'un concentré de protéines de féverole préparé par extraction alcaline.

\section{Matériel et méthodes}

\section{Aliments}

Quatre concentrés protéiques (Poisson micronisé, Soja fermenté, Soja concentré et Féverole) dont la composition et le mode de préparation sont indiqués dans le tableau $\mathrm{I}$, ont servi à préparer quatre aliments d'allaitement dans lesquels ils apportaient de 73 à $8 \mathrm{I}$ p. Ioo des protéines (tabl. 2). Le reste des matières azotées était fourni par de la poudre de lactosérum et un supplément de méthionine. 


\section{TABLEAU I}

Composition des sources de protéines étudiées

(p. too de la matière sèche)

Composition of the protein concentrates

(p. I00 of dry matter)

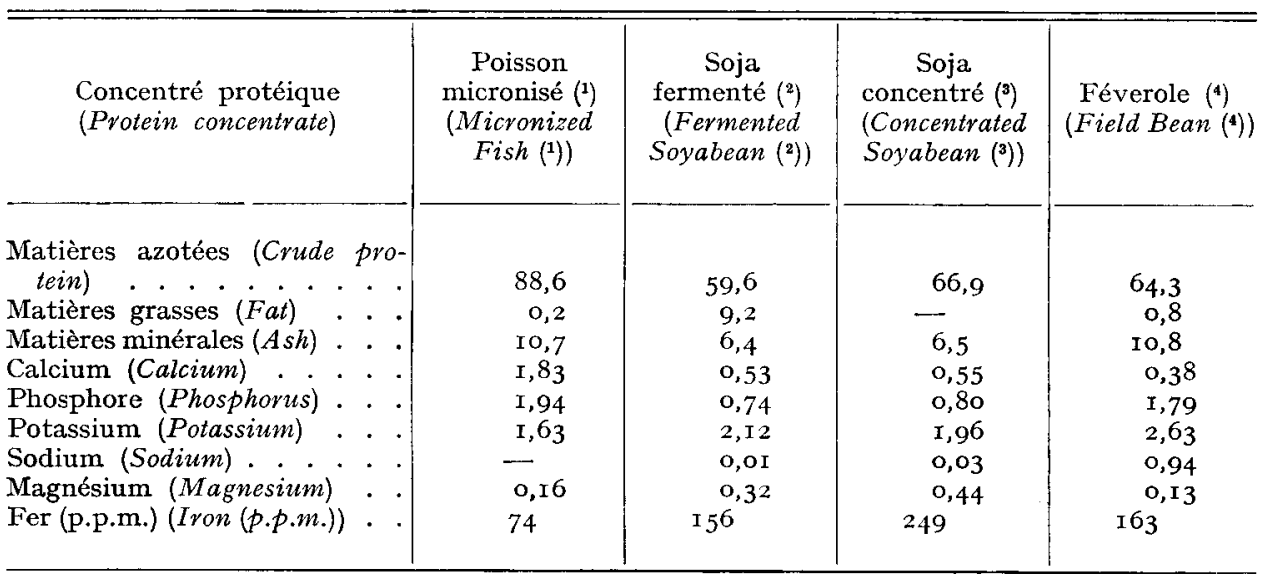

(1) Farine de poisson de Norvège délipidée par extraction à l'hexane et finement broyée (taille moyenne des particules : $35 \mu)$ (NorSILDMEL, 50oI, BERGEN, NORvìge).

(2) Tourteau de soja débarrassé de son saccharose et de ses oligosaccharides (stachyose et raffinose), ainsi que de ses facteurs antitrypsiques par fermentation par Geotrichum candidum (T. STARoN, Iaboratoire des Antibiotiques, I.N.R.A., Chartres et Société Europrotéines, I6, rue d'Inkermann, 79000 Niort).

(3) Tourteau de soja traité par la chaleur humide et débarrassé de son saccharose et de ses oligosaccharides par extraction éthanolique à chaud (HAYPRo-T, HAyes LTD, Ashood, Istaël).

(4) Concentré protéique obtenu à partir de graines de féverole par extraction aqueuse à pH 8,5 , élimination de la majeure partie des constituants insolubles par décantation et séchage par le procédé Spray (Iaboratoire de Technologie Iaitière, I.N.R.A., Rennes). Quatre préparations (A, B, C et D) ont été utilisées respectivement pendant les deux premières périodes, les deux suivantes, la cinquième et la sixième. $A$ et $D$ ont été fabriquées à partir de graines décortiquées, $B$ et $C$ a partir de graines non décortiquées. Lees valeurs rapportées pour la composition sont des moyennes.

(1) Hexane extracted and finely ground fish flour from Norway (mean particle size: $35 \mu$ ) (NorsILDMEL, 500T, Bergen, Norway).

(2) Soya bean oil meal cleared of its sucrose and oligosaccharides (stachyose and raffinose) and antitrypsic factors by Geotrichum candidum fermentation (T. STARON, Laboratoire des Antibiotiques, I.N.R.A., Chartres and Société Europrotéines, I6, rue d'Inkermann, 79000 Niort).

(3) Soya-bean oil meal treated by moist heating and cleared of its sucrose and oligosaccharides by ethanol extraction Haypro-T, Hayes LTD, Ashood, Israel).

(4) Protein concentrate prepared from field bean seeds by aqueous extraction at $p H 8.5$, elimination of the main part of the insoluble constituents by decantation and drying by the Spray process (Laboratoire de Technologie Laitiere, I.N.R.A., Rennes). Four samples (A,B,C and D) were used respectively during the two first, the third and the fourth, the fifth and the sixth period of measure. A and $D$ were made from dehulled seeds, $B$ and $C$ trom whole seeds. The reported composition figures are mean values.

\section{Animaux et régimes}

Les essais ont été effectués sur I 2 veaux mâles de race Frisonne achetés à l'âge d'environ 8 jours et placés dans des cages à bilans dès leur arrivée. Les mesures n'ont commencé qu'une semaine plus tard de façon à laisser les animaux s'adapter à leurs nouvelles conditions d'existence; elles ont alors été effectuées généralement pendant 6 périodes successives de I I jours séparées par des interpériodes de 3 jours. Cependant, pour des raisons matérielles, trois des veaux ont dû être retirés de 
TABLEAU 2

Composition des aliments

Composition of the diets

\begin{tabular}{|c|c|c|c|c|}
\hline $\begin{array}{l}\text { Aliment } \\
(\text { Diet })\end{array}$ & $\begin{array}{c}\text { Poisson } \\
\text { micronisé } \\
\text { (Micronized } \\
\text { Fish) }\end{array}$ & $\begin{array}{c}\text { Soja } \\
\text { fermenté } \\
\text { (Fermented } \\
\text { Soyabean) }\end{array}$ & $\begin{array}{c}\text { Soja } \\
\text { concentré } \\
\text { (Concentrated } \\
\text { Soyabean) }\end{array}$ & $\begin{array}{c}\text { Féverole } \\
(\text { Field Bean) }\end{array}$ \\
\hline 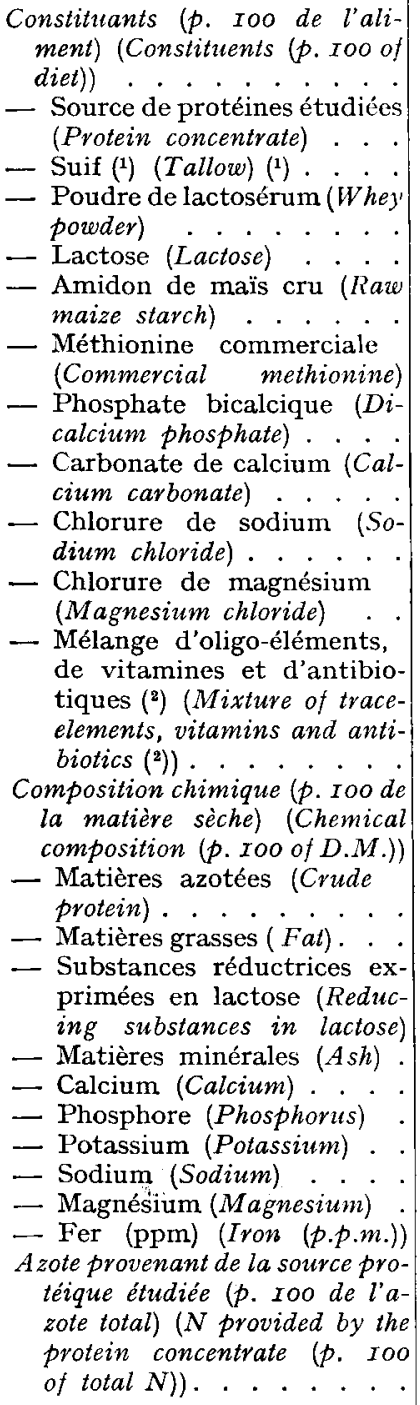 & $\begin{array}{l}24,0 \\
20,0 \\
37,0 \\
13,0 \\
4,0 \\
0,20 \\
- \\
0,60 \\
- \\
0,50\end{array}$ & $\begin{array}{l}35,5 \\
15,7 \\
45,4 \\
\ldots \\
- \\
0,4^{\circ} \\
1,5^{\circ} \\
- \\
0,60 \\
0,20\end{array}$ & $\begin{array}{r}29,0 \\
20,0 \\
36,8 \\
9,7 \\
- \\
0,47 \\
2,00 \\
0,60 \\
0,50 \\
0,20\end{array}$ & $\begin{array}{c}29,3 \\
20,6 \\
47,3 \\
- \\
- \\
0,55 \\
- \\
1,50 \\
- \\
-\end{array}$ \\
\hline
\end{tabular}

(1) Dans tous les aliments, le suif avait été incorporé dans du lactosérum liquide à raison de 35 p. Ioo de la matière sèche. Le mélange ainsi obtenu avait été homogénéisé et séché par le procédé Spray.

( ${ }^{2}$ Pour la composition du mélange de vitamines et d'antibiotiques, of. Paruelle et al., (I972). En outre, les oligoéléments (cuivre, mangan ¿se, zinc, cobalt) ont été ajoutés en proportions variables selon la composition des matières premières.

(1) In each diet, tallow was mixed in liquid whey at the level of 3.5 p. 10o of dry matter. This mixture was homogenized and dried by the Spray process.

(2) For the vitamins and antibiotics mixture composition, see PARUELLE et al. (1972). The trace-elements (copper, manganese, zinc, cobalt) were added in a variable amount according to the raw material composition. 
l'essai, l'un pendant la quatrième période (aliment Soja concentré) et les deux autres à l'issue de la cinquième période (aliments Soja fermenté et Féverole).

Pendant les quatre premiers jours qui ont suivi leur arrivée, les animaux ont tous reçu un lait de remplacement riche en lait écrémé auquel a été progressivement substitué en 3 jours un des aliments expérimentaux. Ces aliments ont été distribués à 3 animaux par régime. Les veaux étaient alimentés au seau, en fonction de leur poids métabolique (poids vif 0,75 ) à raison de 2 repas par jour, suivant le plan de rationnement proposé par PARUELle et al. (I972).

\section{Mesures}

Les veaux étaient pesés à jeun le matin du premier jour de chaque période et le lendemain de la fin de la période. Les quantités refusées ont été pesées après chaque repas et ont servi à constituer des échantillons moyens par veau et par période, qui ont été conservés à $-I_{5}{ }^{\circ} \mathrm{C}$ avant d'être lyophilisés. Les fèces et les urines de chaque veau ont été recueillies séparément et pesées tous les jours. Une partie aliquote des fèces a été séchée à $80^{\circ} \mathrm{C}$ pendant 24 heures dans une étuve à circulation d'air chaud en vue de déterminer la teneur journalière en matière sèche; l'autre partie a servi à constituer des échantillons moyens par veau et par période qui ont été traités comme les échantillons de refus. Une partie aliquote des urines a servi à constituer des échantillons moyens par veau et par période qui ont été conservés à $-I 5^{\circ} \mathrm{C}$.

\section{Méthodes d'analyses}

La teneur en matière sèche des échantillons d'aliments, de refus et de fèces lyophilisés a été déterminée par séchage à roo ${ }^{\circ} \mathrm{C}$ pendant 5 heures.| La teneur en cendres a été obtenue après incinération au four à $55^{\circ}{ }^{\circ} \mathrm{C}$ pendant 6 heures. Le dosage des sucres réducteurs a été effectué par la méthode de Somogy (I952) et celui de l'azote par la méthode de KJELDAHL. Celui des matières grasses a été réalisé sur les aliments et les refus selon la méthode acido-butyrométrique de GERBER, et sur les fèces par extraction à l'éther éthylique après hydrolyse acide. L'interprétation statistique a été effectuée à l'aide de l'analyse de variance à quatre moyennes et de la méthode des contrastes de Scheffe (I959).

\section{Résultats}

Croissance et quantités ingérées (tabl. 3)

Les gains de poids vif ont été satisfaisants avec l'aliment Soja concentré, peu élevés avec les aliments Poisson micronisé et Soja fermenté et très faibles avec l'aliment Féverole.

Les aliments ont été généralement bien consommés pendant toute la durée 
TABLEAU 3

Croissance et efficacité alimentaire (moyennes et écarts-types des échantillons)

Growth and feed conversion rate (means and standard errors of samples)

\begin{tabular}{|c|c|c|}
\hline$\underset{(\text { Diet })}{\text { Aliment }}$ & $\begin{array}{l}\text { Gain de poids vif }(\mathrm{g} / \mathrm{j}) \\
(\text { Live weight gain }(g / d))\end{array}$ & $\begin{array}{c}\text { Matière sèche ingérée } / \mathrm{kg} \\
\text { de gain de poids vif (kg) } \\
(D M \text { intake } / \text { kg live weight } \\
\text { gain }(k g))\end{array}$ \\
\hline $\begin{array}{r}\text { Poisson micronisé (Micronized } \\
\text { Fish) } \\
\end{array}$ & $914\left(\mathrm{r}_{7} 8\right)$ & I.777 (o, I 29) \\
\hline $\begin{array}{c}\text { Soja fermenté (Fermented Soya- } \\
\text { bean) } \ldots . . .\end{array}$ & $902 \quad(40)$ & $\mathrm{I}, 854(0,07 \mathrm{I})$ \\
\hline $\begin{array}{c}\text { Soja concentré (Concentrated } \\
\text { Soyabean) } . . . . . . .\end{array}$ & $1086 \quad(57)$ & $1,702(0,167)$ \\
\hline Féverole (Field Bean). . . . . & $535 \quad(85)$ & $2,463(0,295)$ \\
\hline
\end{tabular}

de l'expérience à l'exception de l'aliment Féverole. Les refus alimentaires ont été respectivement de 3,6 et $2 \mathrm{p}$. Ioo des quantités proposées chez les veaux recevant les aliments Soja concentré, Poisson micronisé et Soja fermenté. Pendant les deux premières périodes, l'aliment Féverole a été très bien accepté par les animaux (3 p. Ioo seulement des quantités proposées ont été refusées). En revanche, pendant les périodes suivantes, les refus ont été très importants (24 p. IOO).

L'efficacité alimentaire a été peu satisfaisante avec les aliments Poisson micronisé, Soja concentré et Soja fermenté, et a été très médiocre avec l'aliment Féverole.

Diarrhées et état sanitaire (tabl. 4)

Pendant toute la durée de l'expérience, l'état sanitaire a été satisfaisant et la fréquence des jours de diarrhées a été très faible pour les aliments Soja concentré, Soja fermenté et Poisson micronisé (respectivement 0,5 et 5 p. Ioo). En revanche, avec l'aliment Féverole, l'état sanitaire des animaux a été médiocre et la fréquence des jours de diarrhées a été importante, surtout en fin d'expérience où elle a atteint $50 \mathrm{p}$. 100.

\section{Digestibilité des aliments (fig. $\mathrm{I}$ et tabl. 5)}

Le coefficient d'utilisation digestive apparente des matières azotées a varié d'une part avec l'âge des animaux, d'autre part avec la nature des matières azotées alimentaires. Il a été moins élevé au cours de la première période de mesures chez les veaux ayant reçu les aliments Poisson micronisé, Soja fermenté et Féverole 
TABLEAU 4

État des fèces

Fecal consistency

\begin{tabular}{|c|c|c|c|}
\hline \multirow{2}{*}{$\begin{array}{l}\text { Aliment } \\
(\text { Diet })\end{array}$} & \multicolumn{3}{|c|}{$\begin{array}{l}\text { État des fèces (p. Ioo du nombre de jours de récolte (1) } \\
(\text { Fecal consistency }(p \text {. Ioo of sampling days)) (1) }\end{array}$} \\
\hline & $\begin{array}{l}\text { diarrhéique } \\
\text { (diarrhoeal) }\end{array}$ & $\begin{array}{l}\text { relâché } \\
\text { (loose) }\end{array}$ & $\begin{array}{c}\text { normal } \\
(\text { normal })\end{array}$ \\
\hline Poisson micronisé (Micronized Fish) . . & $5, \mathrm{I}$ & 79,7 & I 5,2 \\
\hline Soja fermenté (Fermented Soyabean) . . . & 5,0 & 67.7 & 27,3 \\
\hline Soja concentré (Concentrated Soyabean) & $\circ$ & 89,8 & 10,2 \\
\hline 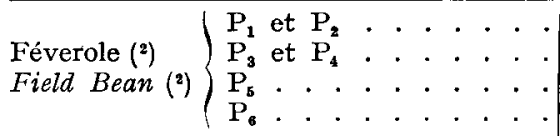 & $\begin{array}{l}\text { II,I } \\
0 \\
40,0 \\
50,0\end{array}$ & $\begin{array}{l}68,5 \\
6 \mathrm{r}, 7 \\
43,3 \\
50,0\end{array}$ & $\begin{array}{c}20,4 \\
38,3 \\
16,7 \\
0\end{array}$ \\
\hline
\end{tabular}

(1) L'état des fèces a été caractérisé d'après leur teneur en matière sèche : moins de I 2 p. Ioo : diarrhéique; entre 12 et $20 \mathrm{p}$. Ioo : relâché; plus de $20 \mathrm{p}$. roo : normal (BLAxTER et WOOD, I953).

(2) $\mathbf{P}_{\mathbf{n}}=n^{\text {ieme }}$ période de mesures.

(1) The feces were classified according to their dry matter content: less than I2 p. Ioo: diarrhoeal; between I2 and 20 p. 100 : loose; more than 20 p. 100 : normal (BLAxTER and Wood, I953).

(2) $P_{n}:$ ith period of measure.

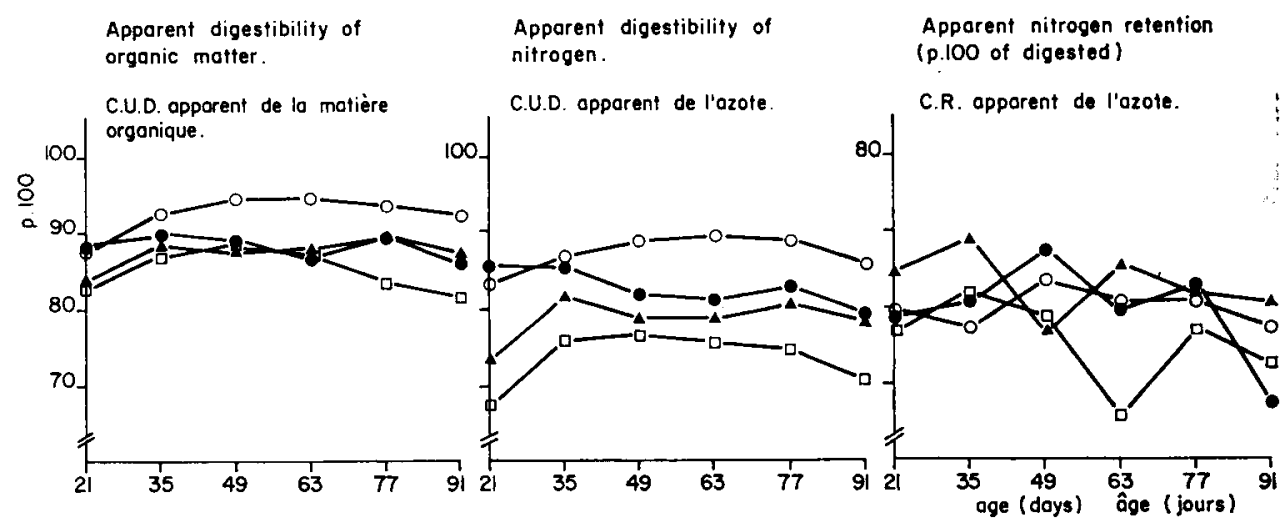

I'IG. I. - Evolution des coefficients de digestibilité apparente de la matière organique et de l'azote, et du coefficient de rétention apparente de l'azote avec l'âge.

- Evolution with age of the apparent digestibility of nutrients and the apparent nitrogen retention.

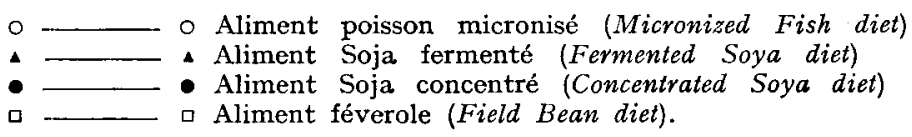


TABLEAU 5

Utilisation digestive et rétention azotée (moyennes et écarts types des échantillons)

Apparent digestibility and nitrogen retention (means and standard-errors of samples)

\begin{tabular}{|c|c|c|c|c|}
\hline $\begin{array}{c}\text { Aliment } \\
(\text { Diet })\end{array}$ & $\begin{array}{c}\text { Poisson } \\
\text { micronisé } \\
\text { (Micronized } \\
\text { Fish) }\end{array}$ & $\begin{array}{c}\text { Soja } \\
\text { fermenté } \\
\text { (Fermented } \\
\text { Soyabean })\end{array}$ & $\begin{array}{c}\text { Soja } \\
\text { concentré } \\
\text { (Concentrated } \\
\text { Soyabean) }\end{array}$ & $\begin{array}{c}\text { Féverole } \\
\text { (Field Bean })\end{array}$ \\
\hline 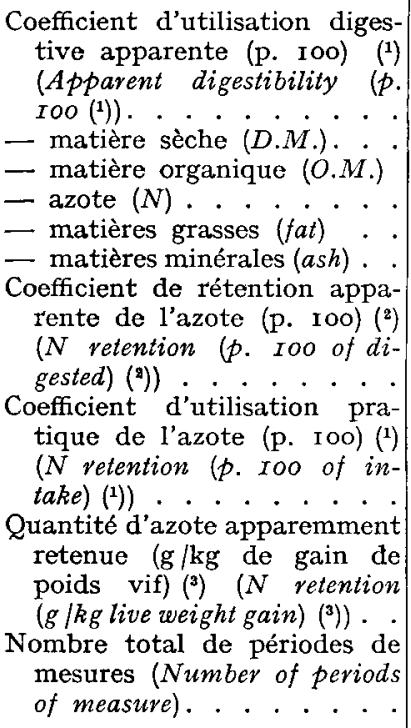 & $\begin{array}{ll}92,0 & (2,7) \\
92,6 & (2,7) \\
85,5 & (4,3) \\
85,6 & (5,6) \\
82,7 & (4,6)\end{array}$ & $\begin{array}{ll}88, I & (2.4) \\
88,5 & (2,4) \\
80,0 & (4, I) \\
8 \mathrm{I}, 0 & (4, I) \\
83,4 & (3,2)\end{array}$ & $\begin{array}{ll}88,0 & (2,0) \\
88,9 & (1,9) \\
82,8 & (4,3) \\
85,7 & (3, \mathrm{I}) \\
75,6 & (4,7)\end{array}$ & $\begin{array}{rr}84,5 & (3,3) \\
85,8 & (3,7) \\
74,9 & (4,6) \\
74, \mathrm{I} & (\mathrm{I} \mathrm{I}, 6) \\
70,0 & (4,9)\end{array}$ \\
\hline
\end{tabular}

(1) Moyennes et écarts types en excluant la première période de mesures.

$\left({ }^{2}\right)$ Moyennes et écarts types de toutes les périodes de mesures.

(3) Pendant la durée totale de l'expérience.

(1) Means and standard errors without the first pcriod of measure.

(3) Means and standard errors zeith all the periods of measure.

(3) During the whole trial.

(respectivement 79,3-73,6 et 67,5 p. roo) puis il a augmenté et s'est maintenu relativement constant sauf au cours de la dernière période où il a légèrement baissé. En revanche, avec l'aliment Soja concentré, il a eu tendance à diminuer pendant toute la durée de l'expérience (fig. I).

Au delà du premier mois d'âge, l'utilisation digestive des matières azotées a été satisfaisante pour l'aliment Poisson micronisé (85,5 p. I0o); elle a été moins élevée pour les aliments Soja concentré (82,8 p. IOo), Soja fermenté (80,0 p. IOo) et surtout Féverole (74,9 p. roo). Les différences observées entre l'aliment Féverole et les trois autres aliments sont significatives $(p<0,05)$; il en est de même entre les aliments Poisson micronisé et Soja fermenté $(\mathrm{p}<0,05)$. En supposant 
que la digestibilité des protéines apportées dans ces aliments par le lactosérum est la même que celle des protéines du lactosérum partiellement délactosé par ultrafiltration (94,3 p. Ioo d'après Toullec et al., I974), les valeurs estimées pour la digestibilité apparente des protéines du Poisson micronisé, du Soja fermenté, du Soja concentré et de la Féverole sont alors respectivement de $83,76,80$ et 67 p. Iоo.

La digestibilité apparente de la matière organique, des matières grasses et des matières minérales des aliments a varié avec l'âge des animaux et la nature de l'aliment, parallèlement à celle des matières azotées (tabl. 5 et fig. I). Au cours de la première période de mesures, elle a toujours été inférieure. Par la suite, le CUD apparent de la matière organique a été plus élevé pour l'aliment Poisson micronisé $(92,6$ p. IOO) que pour les aliments Soja concentré $(88,9$ p. Ioo), Soja fermenté $(88,5$ p. Ioo) et surtout Féverole $(85,8$ p. I00). Toutefois, seule la valeur obtenue pour l'aliment Poisson micronisé diffère statistiquement des trois autres $(\mathrm{P}<0,05)$.

\section{Rétention azotée}

Le coefficient de rétention apparente de l'azote a varié irrégulièrement avec l'âge des animaux (fig. I) mais il a été satisfaisant avec tous les régimes (55 à 63 p. roo) (tabl. 5). Les valeurs les plus élevées ont été obtenues avec les aliments Soja concentré et Soja fermenté et la moins forte avec l'aliment Féverole. La différence observée entre les aliments Soja fermenté et Féverole est significative $(\mathrm{p}<0,05)$.

Le coefficient d'utilisation pratique de l'azote a été semblable (50 à 54 p. Ioo) pour les aliments Poisson micronisé, Soja fermenté et Soja concentré (tabl. 5). Ce coefficient a été plus faible avec l'aliment Féverole (40 p. I00), en raison de sa digestibilité inférieure. La quantité d'azote apparemment retenue par $\mathrm{kg}$ de gain de poids vif a été anormalement élevée avec l'aliment Féverole (43 g au lieu de 34 à 38 avec les autres aliments); cela est peut-être dû à des pertes d'urine, ainsi qu'à une plus grande imprécision dans les mesures, du fait du gain de poids vif très faible des animaux qui recevaient l'aliment Féverole.

\section{État d'anémie des animaux}

La viande des animaux était un peu trop colorée avec les aliments Soja concentré et Poisson micronisé. Les animaux qui ont reçu les deux autres aliments n'ont pas été abattus en fin d'expérience. Les valeurs moyennes de l'hématocrite à la fin de la sixième période de mesures ont été respectivement de $27,28,28$ et 24 pour les veaux qui ont reçu les aliments Poisson micronisé, Soja fermenté, Soja concentré et Féverole. Ces résultats sont à rapprocher des teneurs en fer relativement élevées des aliments (tabl, 2). 


\section{Discussion}

Les résultats de cet essai s'accordent avec la plupart des observations générales effectuées lors de nos précédents travaux sur la digestibilité des matières azotées chez le veau préruminant (Toullec et al., Ig69; PARUelle et al., I972; Toullec, Mathieu et Pion, I974; Toullec, Frantzen et Mathieu, I974; TOULLEC et al., I974). En particulier, ils confirment l'utilisation digestive moins élevée de la plupart des protéines de remplacement par rapport à celles du lait (95 p. I0O); les différences atteignent respectivement I2, I5, I9 et 28 points pour le Poisson micronisé, le Soja concentré, le Soja fermenté et la Féverole.

La digestibilité semble fortement influencée par la présence de constituants peu digestibles dans les concentrés protéiques; ainsi, la valeur la plus élevée est obtenue pour le Poisson micronisé qui ne contient pas de glucides complexes. De même, les deux concentrés protéiques de soja sont un peu mieux utilisés qu'un tourteau de soja cuit (dont les protéines ont un CUD apparent de 74 p. Ioo, d'après Paruelle et al., 1972); cela est probablement dû à l'élimination quasi-totale du saccharose et des $\alpha$ galactosides lors de l'extraction alcoolique et de la fermentation (BEsLE et Pitior, 1976). Ces oligosides semblent favoriser le développement de fermentations microbiennes dans le cœcum et le côlon du veau (BEsLE, Brion et Thivend, résultats non publiés). Cependant, l'amélioration que nous observons ( 2 à 6 points) est beaucoup plus faible que celle ( 13 points) obtenue par Porter et Hill (I963) en éliminant également les autres glucides du soja (cellulose, hémicelluloses...). Nos concentrés protéiques de Féverole étaient dépourvus de cellulose même quand ils avaient été préparés à partir de graines entières; en revanche, ils contenaient probablement des proportions importantes d'oligosides qui constituent environ 6 p. Ioo de la matière sèche des cotylédons (Cerning, Saposnik et Guilbot, 1975) et sont solubles dans l'eau.

La présence de facteurs antitrypsiques peut aussi avoir eu un effet dépressif sur la digestibilité des protéines végétales que nous avons étudiées. Ces substances existent dans la Féverole (Wilson, McNab et Bentley, 1972). Elles subsisteraient en partie sous forme liée dans les concentrés protéiques de Soja, malgré les traitements thermiques subis. Cette fraction pourrait encore être libérée par un traitement acide (Delobez, Dutertre et Rambaud, I97I), alcalin (RAmSeY et Williard, 1975) ou enzymatique (Wang, Vespa et Hesseltine, 1972) et être ensuite détruite par la chaleur.

Néanmoins, l'absence de glucides complexes et de facteurs antitrypsiques ne suffit pas pour obtenir une digestibilité aussi élevée pour les protéines de remplacement que pour celles du lait, comme le montrent les résultats observés avec le Poisson micronisé. D'autres traitements technologiques, tels qu'une hydrolyse enzymatique partielle, pourraient améliorer la digestibilité : les protéines de poissons blancs ainsi traitées ont un CUD apparent de 9I p. Ioo (Paruelle et al., 1974).

D'ailleurs, les écarts observés dans la digestibilité entre les protéines pourraient peut-être s'expliquer en partie par des différences dans leur digestion au niveau de la caillette (GuIlloteau et al., I975 et I976). Ainsi, par rapport à l'in- 
gestion d'un aliment classique, riche en protéines de lait, celle de l'aliment Poisson micronisé entraîne, au cours des premières heures après le repas, un affluxplus important dans le duodénum d'azote total et d'azote protéique. Ce phénomène paraît plus accentué avec l'aliment Soja concentré et surtout avec l'aliment Féverole. En outre, l'ingestion des aliments Poisson micronisé, Soja concentré, Soja fermenté et surtout Féverole semble provoquer un accroissement des quantités d'azote protidique d'origine endogène et/ou microbienne excrété dans les fèces; celles d'origine alimentaire paraissent davantage augmentées avec les aliments Soja fermenté et Féverole qu'avec les aliments Soja concentré et Poisson micronisé (Patureau-Mirand et al., I976).

La médiocrité des résultats zootechniques obtenus avec l'aliment Féverole a peut-être été causée également par d'autres substances indésirables, ainsi, les aglycons de la vicine et de la convicine et la 3-4-dihydroxyphénylalanine, ont été trouvés dans la fève et sont considérés comme responsables du favisme chez l'homme (MAGER, Razin et Hershko, I969). La Féverole contient également des hémaglutinines (MARQUARDT et al., I974) et des tanins, ces derniers étant localisés principalement dans les téguments (TANGUY et MarTin, I973). Cependant, nous n'avons pas observé d'effet systématique du décorticage préalable de la graine avant l'extraction. La formation de lysino-alanine, toxique chez le rat, a été signalée lors de l'extraction alcaline des protéines de Soja (WOODARD et SHORT, r973); elle n'a cependant pas été observée dans le cas de la Féverole (BAUdeT et Mosse, I973). Par ailleurs, l'aliment Féverole était particulièrement riche en matières minérales, surtout en potassium et en sodium (tabl. 2), auxquels un effet laxatif a été attribué par BusH et al., (I963).

Pour être bien utilisées, les protéines de la Féverole devraient donc subir des traitements technologiques mieux appropriés. Ainsi, Wilson et McNAB (I972), VERMOREL (I973) et LE Dividich (I973) ont amélioré les résultats obtenus chez le poulet et le rat, en soumettant la Féverole à des traitements thermiques. Au cours d'un essai préliminaire sur le veau, nous avons également observé un accroissement de l'appétibilité d'un concentré protéique de fève préparé par turboséparation, en le chauffant en présence de vapeur d'eau à Ioz ${ }^{\circ} \mathrm{C}$, pendant Io minutes.

Par ailleurs, plusieurs auteurs ont mis en évidence des anticorps spécifiques des protéines alimentaires dans le sang de veaux recevant des protéines de Soja (Van Adrichem et Frens, r965; Van Leeuwen, Weide et BraAs, i969; Smith et Sissons, I975). Chez nos animaux, Coleno (résultats non publiés) a effectué les mêmes observations avec le Soja concentré, le Soja fermenté et la Féverole, mais pas avec le Poisson micronisé.

En conclusion, les concentrés protéiques de Poisson et de Soja étudiés pourraient être introduits dans les aliments d'allaitement pour veaux. Des essais complémentaires seraient cependant nécessaires pour préciser les taux pratiques d'incorporation. En revanche, il n'en serait pas de même pour le concentré de Féverole, sans une amélioration de sa technologie de préparation. 


\title{
Remerciements
}

A. M. Berthelem, de la Station d'Amélioration des Plantes de l'I.N.R.A. (Centre de Rennes), qui nous a fourni la féverole (lignée $29 \mathrm{E}$ ) utilisée dans cette étude et à Madeleine DouAIRE de la Chaire de Zootechnie (E.N.S.A. de Rennes), qui nous a conseillé dans l'analyse statistique des résultats.

\section{Summary}

\author{
Utilization of proteins by the preruminant veal calf. \\ V. - Apparent digestibility of fish, soyabean, and field bean proteins
}

The object of this work was to study the apparent digestibility of different proteins used as a substitute for milk proteins in milk replacers for veal calves.

Four protein concentrates were used (table I), i.e. Micronized fish, a hexane extracted and finely ground fish fluor from Norway, Fermented soya-bean, a soya-bean oil meal cleared of its oligosaccharides by Geotrichum candidum fermentation, concentrated soya-bean, a soya-bean oil meal treated by moist heating and cleared of its oligosaccharides by ethanol extraction, Field bean, a protein concentrate prepared from field bean seeds by aqueous extraction at $\mathrm{pH} 8.5$. Four milk replacers were prepared with these four protein concentrates supplying $81,77,78$ and $73 \mathrm{p}$. cent, respectively of crude protein, the rest being provided by whey and a methionine supplement (table 2).

Tweive male calves ( 3 per diet) were used. They were kept in balance crates between the ages of about 8 and 54-96 days. The diets were well consumed, except that containing fieldbeans, at the end of the experiment. Growth and feed efficiency were much poorer with the field-bean diet than with the others (table 3 ). The frequency of days with diarrhoea was low in all cases except for field-beans, and more particularly at the end of the experiment (table 4).

The apparent digestibility of crude protein increased by 5 to 9 points during the first month of life of the animals (except for the concentrated soyabean diet) (fig. I). Afterwards, it was the highest for the micronized fish diet $(85.5 \mathrm{p}$. cent), less satisfactory for the concentrated soyabean diet $(82.8 \mathrm{p}$. cent) and fermented soya-bean diet (80.0 p. cent) and very low for the field bean diet $(74.9 \mathrm{p}$. cent). Values estimated for protein digestibility of micronized fish, concentrated soya-bean, fermented soya-bean and field-bean were $83,80,76$ and $67 \mathrm{p}$. cent respectively. Retention of apparently absorbed nitrogen was satisfactory for all diets, but less high however with the field-bean diet.

According to these results and provided that further trials are performed the fish and soyabean concentrates studied might be incorporated in large amounts into the milk replacers. But, this would not be possible with the field-bean concentrate without improving its technological processing.

\section{Références bibliographiques}

Batdet J., Mosse J., I973. (Communication personnelle).

Besle J. M., Pitrot M., 1976. Extraction et purification des glucides : Application à divers aliments dérivés du soja. Ann. Biol. anim. Bioch. Biophys., 16, 753-772.

Blaxter K. L., Wood W. A., 1953. Some observations on the biochemical and physiological events associated with diarrhoea in calves. Vet. Rec., 50, 889-892.

Cerning J., Saposnik A., Guilbot A., 1975. Carbohydrate compasition of horse beans (Vicia faba) of different origins. Cereal Chemist., 52, I25-137.

Delobez R., Dutertre R., Rambaud M., i97I. Dosage des facteurs antitrypsiques du soja. Rev. Fr. Corps Gras, 18, 38I-389.

Duthie I. F., Edwards D. G., Rogers B., Andrews R. J., Wright J. A., 1974. Preliminary studies on the suitability of field bean (Vicia faba L.) protein isolate for lambs and calves. Proc. Nutr. Soc., 33, 4OA-4 IA.

Frantzen J.-F., Toullec R., Thivend P., Mathieu C.-M., 1973. Influence de la coagulation des protéines sur la vidange stomacale chez le veau préruminant. Ann. Biol. anim. Bioch. Biophys., 13, 699-796. 
Gorrill A. D. L., Thomas J. W., 1967. Body weiglt changes, pancreas size and enzyme activity, and proteolytic enzyme activity and protein digestion in intestinal contents from calves fed soy-bean and milk protein diets. $J$. Nutr., 92, 21 5-223.

Gorrill A. D. L., Thomas J. W., Stewart W. E., Morrill J. L., I967. Exocrino pancreatic secretion by calves fed soy-bean and milk protein diets. $J$. Nutr., 92, 86-92.

Guilloteau P., Paruelle J.-L., Toullec R., Mathieu C.-M., I975. Utilisation des protéines par le veau préruminant à l'engrais. III. - Influence du remplacement des protéines du lait par celles du poisson sur la vidange stomacale. Ann. Zootech., 24, 243-253.

Guilloteau P., Paruelle J.-L., Toullec R., Mathieu C.-M., i976. Utilisation des protéines par le veau préruminant à l'engrais. VI. Influence du remplacement des protéines du lait par celles du soja et de la féverole sur la vidange stomacale. (En préparation).

Lassister C. A., Fries G. F., Huffman C. F., Duncan C. W., 1959. Effect of pepsin on the growth and health of young dairy calves fed various milk replacers rations. J. Dairy Sci., 42, 666-67o.

LE Drvidich J., 1973. Valeur protidique des graines de Vigna sinensis et de féverole: comparaison chez le rat avec le tourteau de soja. Ann. Zootech., 22, 267-277.

Mager J., Razin A., Hershko A., 1969. Favism. In Liener I. E., Toxic constituents of plant foodstuffs, 293-318, Academic Press, New-York.

Marquardi R. R., Campbell L. D., Stothers S. C., McKirdy J. A., I974. Growth responses of ducks and rats fed diets containing four cultivars of raw or autoclaved faba beans. Can. J. Anim. Sci., 54, I77-182.

Nitsan Z., Volcani R., Gordin S., Hasdai A., r97r. Growth and nutrient utilization by calves fed milk replacers containing milk or soybean protein concentrate heated to various degrees. J. Dairy Sci., 54, 1294-1299.

Noller C. J., Ward C. M., MacGilliard A. D., Huffman C. F., Duncan C. W., I956. The effect of age of the calf on the availability of nutrients in vegetable milk-replacer ration. $J$. Dairy Sci., 39, I288-1298.

Paruelle J.-L., Toullec R., Frantzen J.-F., Mathieu C.-M., 1972. Utilisation des protéines par le veau préruminant à l'engrais. I. Utilisation digestive des protéines de soja et des levures d'alcanes incorporées dans les aliments d'allaitement. Ann. Zootech., 21, 3I9-33I.

Paruelle J.-L., Toullec R., Patureau-Mirand P., Mathieu C.-M., I974. Utilisation des protéines par le veau préruminant à l'engrais. II. Utilisation des protéines de poisson et influence de l'addition d'un complexant du fer. Ann. Zootech., 23, 519-536.

Patureau-Mirand P., Touliec R., Guilloteau P., Pion R., I977. Influence de la nature des protéines alimentaires sur la composition en acides aminés des fèces du veau préruminant. Ann. Biol. anim. Bioch. Biophy., 17, 7 I-83.

Pron R., I97r. Composition en acides aminés des aliments. Ind. Alim. Anim., B, 29-3 .

Porter J. W. G., Hill W. B., I963. Nitrogen balance trials with calves given synthetic milk diets. Nat. Inst. For Research in Dairying. Annual report. I 26.

RAmsey H. A., Williard T. R., 1975. Soy protein for milk replacers. J. Dairy Sci., 58, 436-44I.

SCHEFFE H., I959. The analysis of variance, 68-72, Wiley, New-York.

Shoptaw L., t936. Soybean flour as a substitute for cow's milk in feeding dairy calves. $J$. Dairy Sci., 49, 95-99.

Smith R. H., Sissons J. W., I975. The effect of different feeds, including soya-bean products, on the passage of digesta from the abomasum of the preruminant calf. Brit. J. Nutr., 33, 329349.

SOMOGyi M., I952. Notes on sugar determination. J. Biol. Chem., 195, 19-23.

Tanguy J., Martin M., I973. (Communication personnelle.)

Terroine E., I931. De l'emploi des laits artificiels dans l'élevage du bétail. Bull. Soc. Sci. Hyg. Alim. Al. Ration. Homme, 19, I-23.

Toullec R., Frantzen J.-F., Mathieu C.-M., 1974. Influence de la coagulation des protéines du lait sur l'utilisation digestive d'un lait de remplacement par le veau préruminant. Ann. Zootech., 23, 359-364.

Toullec R., Frantzen J.-F., Maubois J.-L., Pion R., 1974. Utilisation digestive par le veau préruminant des protéines du lactosérum traitées par ultrafiltration sur membrane. Techn. Lait., 828, I 7-2 I.

Toullec R., Mathieu C.-M., Pion R., 1974. Utilisation des protéines du lactosérum par le veau préruminant à l'engrais. II. Digestibilité et utilisation pour la croissance. Ann. Zootech., 23, $75-87$. 
Toullec R., Mathinu C.-M., Vassal L., Pion R., ig69. Utilisation digestive des protéines du lactosérum par le veau préruminant à l'engrais. Ann. Biol. anim. Bioch. Biophys., 9, 66I-664.

Toullec R., Thivend P., Mathieu C.-M., I97I. Utilisation des protéines du lactosérum par le veau préruminant. I. Vidange stomacale comparée du lait entier et de deux laits de remplacement ne contenant que des protéines de lactosérum comme source de matières azotées. Ann. Biol. anim. Bioch. Biophys., 11, 435-453.

VAn Adrichem P. W. M., Frens A. M., I965. Soy bean protein as alimentary antigen in fattening calves. Tijdschy. Dievgeneesk., 90, 525-530.

Van Hellemond K. K., 1967, cité par Norrman E., 197I. Fish protein concentrate in milk replacers. Proc. Int. Milk Replacer Symp. Zurich 26-27 May, I4r-I $4^{2}$.

Van Leeuwen V. M., Weide H. J., Braas C. C., 1969. Feeding value of soybean oil meal compared with dried skimmed milk. Versl. Landbouwk. Onderzock. Ned., 732, I-I9.

VAN WEERdEN E. J., 1974. Low fat fish meal in milk replacers. 3rd European Symposium on the use of fish meal in animal feeding, Amsterdam inth-I2th September, 96-Iog. IAFMM, Potters-Bar, Herts., England.

Van Weerden E. J., cité par Norsildmel. Concentré micronisé de protéines pour laits de remplacement. BP I034, Bergen, Norvège.

VERMOREL M., I973. Influence du décorticage sur la valeur nutritive de la féverole. Premiers résultats obtenus chez le porc et le rat en croissance. Bull. Tech. C.R.Z.V., 11, I5-I8.

Wang H. L., Vespa J. B., Hesseltine C. W., 1972. Release of bound trypsin inhibitors in soybeans by Rhizopus oligosporus. $J$. Nutr., 102, I 495-I 499.

Williams J. B., KNodr C. B., I950. The further development of milk replacement for dairy calves. J. Dairy Sci., 33, 809-814.

WiLSON B. J., MCNAB J. M., I972. The effect of autoclaving and methionine supplementation on the growth of chicks given diets containing field beans (Vicia faba L.). Brit. Poul. Sci., 13, 67-73.

Wilson B. J., McNab J. M., Bentley H., I972. Trypsin inhibitor activity in the field bean (Vicia faba L.). J. Sci. Fd. Agric., 23, 679-684.

WOOdARD J. C., ShORT D. O., 1973. Toxicity of alkali-treated soyprotein in rats. J. Nutr., 103, 569-574. 